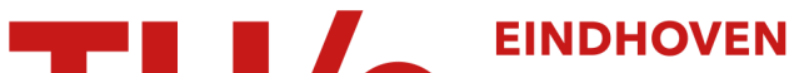 \\ UNIVERSITY OF \\ TECHNOLOGY
}

\section{Selection of sensors and actuators based on a sufficient condition for robust performance}

\section{Citation for published version (APA):}

Wal, van de, M. M. J., \& de Jager, B. (1997). Selection of sensors and actuators based on a sufficient condition for robust performance. In ECC '97: European control conference, conference proceedings, 1-4 July 1997 Belware.

Document status and date:

Published: 01/01/1997

\section{Document Version:}

Publisher's PDF, also known as Version of Record (includes final page, issue and volume numbers)

\section{Please check the document version of this publication:}

- A submitted manuscript is the version of the article upon submission and before peer-review. There can be important differences between the submitted version and the official published version of record. People interested in the research are advised to contact the author for the final version of the publication, or visit the $\mathrm{DOI}$ to the publisher's website.

- The final author version and the galley proof are versions of the publication after peer review.

- The final published version features the final layout of the paper including the volume, issue and page numbers.

Link to publication

\section{General rights}

Copyright and moral rights for the publications made accessible in the public portal are retained by the authors and/or other copyright owners and it is a condition of accessing publications that users recognise and abide by the legal requirements associated with these rights.

- Users may download and print one copy of any publication from the public portal for the purpose of private study or research.

- You may not further distribute the material or use it for any profit-making activity or commercial gain

- You may freely distribute the URL identifying the publication in the public portal.

If the publication is distributed under the terms of Article 25fa of the Dutch Copyright Act, indicated by the "Taverne" license above, please follow below link for the End User Agreement:

www.tue.nl/taverne

Take down policy

If you believe that this document breaches copyright please contact us at:

openaccess@tue.nl

providing details and we will investigate your claim. 


\title{
SELECTION OF SENSORS AND ACTUATORS BASED ON A SUFFICIENT CONDITION FOR ROBUST PERFORMANCE
}

\author{
Marc van de Wal, Bram de Jager \\ Faculty of Mechanical Engineering, Eindhoven University of Technology \\ P.O. Box 513, 5600 MB Eindhoven, The Netherlands \\ Fax: +3140 2461418,Email:M.M.J.v.d.Wal@wfw.wtb.tue.nl
}

\begin{abstract}
A new method for selecting sensors and actuators for linear control system applications is proposed. The goal is to eliminate actuator/sensor combinations for which a controller achieving a desired robust stability or robust performance level cannot be designed. To account for the structured uncertainty block, the plants for all candidate combinations are extended with certain scaling filters. Each combination is subjected to six conditions for existence of a stabilizing controller meeting a required $\mathcal{H}_{\infty}$ norm bound. However, combinations may be incorrectly rejected, since optimality of the scaling filters is not guaranteed. An active suspension control problem is used as an illustrative example.
\end{abstract}

Keywords: robust control, linear systems, automotive.

\section{Introduction}

Preceding controller design, an appropriate number, place, and type of actuators and sensors must be selected. This will be called Input Output (IO) selection, where "input" refers to a manipulated variable and "output" to a measured variable. Compared to modeling and controller design, little attention has been paid to IO selection. Nevertheless, it is of crucial importance. First, the actuator/sensor combination (IO set) may put fundamental limitations on the system's performance. Second, the IO set determines aspects such as the system's complexity, hardware expenses, and maintenance effort. Due to the combinatorial nature of IO selection, the number of "candidate" IO sets may be huge and favorable ones are easily overlooked. So, an efficient and effective IO selection method is desired. A brief survey of methods is given in [10].

Due to inevitable modeling errors, robustness is a major issue in control system design. In this respect, the IO selection goal considered here is to minimize the number of inputs and outputs, subject to the achievement of a desired Robust Performance ( $R P$ ) level. Thus, with the IO set it must be possible to construct a stabilizing controller meeting the performance specifications for a particular class of uncertainties. Such an IO set will be termed "viable." The proposal of an IO selection method aimed at this goal for linear systems is the main contribution of this paper.

In [9], an IO selection method is discussed employing criteria based on nominal performance and robust stability against separate uncertainties, which are properties necessary for RP. Unfortunately, the method can only deal with structured uncertainties and RP in a conservative way. In the present paper, the method from [9] is modified to solve this shortcoming to some extent. This is accomplished by introducing certain scaling filters accounting for structure. After extending the plant corresponding to each IO set with these filters, the modified method essentially checks the same criteria for IO set viability as the original method. IO sets which pass the test are guaranteed to be viable for $\mathrm{RP}$, but they may also be incorrectly rejected, since the scaling filters need not be optimal.

This paper is organized as follows. First, some aspects related with robust control system design are summarized. Second, the IO selection is treated and, third, it is applied to an active suspension control problem for a tractorsemitrailer. The final section provides some directions for further research. A more extensive treatment of both theory and application is given in [8].

\section{Robust Performance}

Finite-dimensional, linear, time-invariant control systems in the standard set-up of Fig. 1 are considered. The generalized plant $G$ not only includes plant data $P$, but also performance specifications and uncertainty characterizations via the filters $V$ and $W . K$ denotes the controller and $M$ the generalized closed-loop. The block $\Delta=\operatorname{diag}\left(\Delta_{u}, \Delta_{p}\right)$ is assumed stable and serves two purposes. First, it extracts uncertainties from the plant model via the (possibly structured) block $\Delta_{u}$. Second, it transforms performance specifications into stability requirements via the unstructured fictitious block $\Delta_{p}$. The following signals play a role: the measured variables $y$ (outputs); the manipulated variables $u$ (inputs); the controlled variables $z^{*}$, which are ideally zero; the exogenous variables $w^{*}$, such as reference signals and disturbances; the signals $p$ and $q$ associated with the uncertainty block $\Delta_{u}$. Finally $w:=\left(\begin{array}{c}p \\ w^{*}\end{array}\right), z:=\left(\begin{array}{c}q \\ z^{*}\end{array}\right)$.

The proposed IO selection is restricted to dynamic uncertainties. Consequently, real parametric uncertainties are treated as dynamic ones, potentially introducing conservatism. Repeated uncertainties are neither considered, so the uncertainty structure related with $\Delta_{u}$ takes the form:

$$
\Delta_{u}:=\left\{\operatorname{diag}\left(\Delta_{u_{1}}, \Delta_{u_{2}}, \ldots, \Delta_{u_{k}}\right): \Delta_{u_{i}} \in \mathcal{R} \mathcal{H}_{\infty}\right\}
$$

with $\mathcal{R} \mathcal{H}_{\infty}$ denoting stable, real-rational, and proper transfer functions. Invoking the unstructured performance block $\Delta_{p}$, an augmented block structure is defined:

$$
\Delta:=\left\{\operatorname{diag}\left(\Delta_{u}, \Delta_{p}\right): \Delta_{u} \in \Delta_{u}, \Delta_{p} \in \mathcal{R} \mathcal{H}_{\infty}\right\} .
$$




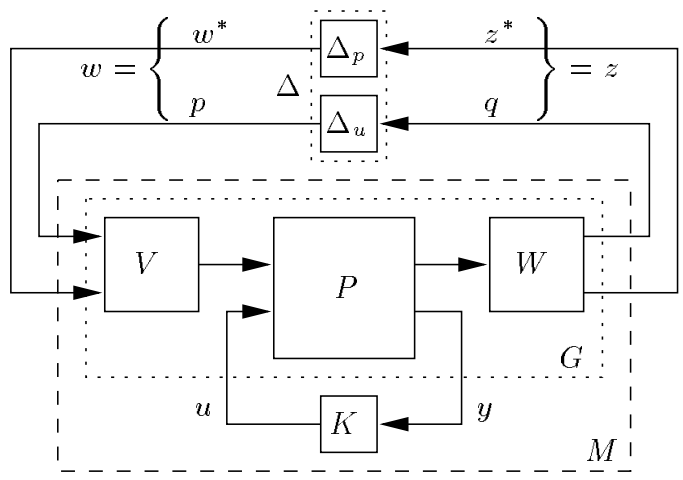

Figure 1: Standard control system set-up

The transfer function from $w^{*}$ to $z^{*}$ is denoted $\tilde{M}=$ $\mathcal{F}_{u}\left(M, \Delta_{u}\right)$. The $\mathcal{H}_{\infty}$ norm of this uncertain closed-loop under the class of uncertainties $\Delta_{u}$ is used as a measure for RP. The following theorem provides a necessary and sufficient condition for an RP level $\gamma$ [11, Chapter 11]:

Robust Performance: Assume $M$ is stable and let $\gamma>0$. For all $\Delta_{u} \in \Delta_{u}$ with $\left\|\Delta_{u}\right\|_{\infty} \leq 1 / \gamma$, the uncertain closedloop $\tilde{M}$ is stable and $\|\tilde{M}\|_{\infty}<\gamma$ if and only if:

$$
\|M\|_{\mu}:=\sup _{\omega} \mu_{\Delta}(M(j \omega))<\gamma .
$$

Here, $\mu$ is the structured singular value, which is a function operating on complex matrices, defined as:

$$
\begin{aligned}
& \mu_{\Delta}(M):=\left[\min _{\Delta \in \Delta}(\bar{\sigma}(\Delta): \operatorname{det}(I-M \Delta)=0)\right]^{-1} \\
& \text { and } \mu_{\Delta}(M):=0, \text { if no } \Delta \text { makes } I-M \Delta \text { singular. }
\end{aligned}
$$

For more details on $\mu$, see, e.g., [6]. For a specified frequency grid, $\mu$ can be used for control system analysis and design. In that context, $\|M\|_{\mu}$ is a robust stability measure, since it is the reciprocal of the magnitude of the smallest structured uncertainty, which destabilizes the system. Moreover, the above theorem shows, that by the introduction of the fictitious block $\Delta_{p}$ the RP problem becomes equivalent to a robust stability problem for the augmented block $\Delta$.

Because $\mu$ cannot be computed exactly in an efficient way, upper and lower bounds are used, the latter of which will not be paid attention to here. The upper bound " ||$M \|_{\bar{\mu}}$ " for $\|M\|_{\mu}$ is defined as [6]:

$$
\|M\|_{\bar{\mu}}:=\sup _{\omega} \inf _{D \in D} \bar{\sigma}\left(D_{z}(\omega) M(j \omega) D_{w}^{-1}(\omega)\right) .
$$

Here, $D \in D$ is shorthand for $D_{z} \in D_{\boldsymbol{z}}, D_{w} \in D_{w}$ and $D_{z}$ and $D_{w}$ are the frequency dependent " $D$-scales" with the corresponding sets:

$$
\begin{aligned}
& D_{z}:=\left\{\operatorname{diag}\left(d_{1} I_{m_{1}}, \ldots, d_{l} I_{m_{l}}\right): d_{j} \in \mathbb{R}, d_{j}>0\right\}, \\
& D_{w}:=\left\{\operatorname{diag}\left(d_{1} I_{n_{1}}, \ldots, d_{l} I_{n_{l}}\right): d_{j} \in \mathbb{R}, d_{j}>0\right\} .
\end{aligned}
$$

Note, that $D_{z}$ and $D_{w}$ only differ in dimensions and that each full block $\Delta_{j}$ in $\Delta$ with $m_{j}$ outputs and $n_{j}$ inputs is accompanied by diagonal scaling matrices $d_{j} I_{m_{j}}$ and $d_{j} I_{n_{j}}$. Without loss of generality, the $D$-scales are normalized with respect to the last diagonal matrix corresponding to $\Delta_{p}: d_{l}=1[6]$.

If $\|M\|_{\mu}$ in (3) is replaced by its upper bound $\|M\|_{\bar{\mu}}$, a sufficient condition for RP results. This modified condition is generally tight, i.e., generally $\|M\|_{\bar{\mu}} \approx\|M\|_{\mu}$. Checking
$\|M\|_{\bar{\mu}}<\gamma$ for a given $M$ is referred to as $\mu$-analysis, while constructing $K$ such that $\|M\|_{\bar{\mu}}<\gamma$ is called $\mu$-synthesis. Hence, IO selection aimed at RP could be performed by $\mu$-syntheses for all candidate IO sets, but this brute force approach is only feasible for a manageable number of candidates. To understand the alternative IO selection to be proposed, a commonly used approximate approach to $\mu$ synthesis is outlined first, see, e.g., [11, Chapter 11].

The goal of "optimal" $\mu$-synthesis is expressed as $\min _{K \in \mathcal{K}_{A}}\|M(G, K)\|_{\mu}$, with $\mathcal{K}_{A}$ the set of all stabilizing, real-rational, and proper controllers. This problem is currently unsolved and replaced by the approximate goal:

$$
\min _{K \in \mathcal{K}_{A}} \inf _{\tilde{D} \in \tilde{D}}\left\|\tilde{D}_{z} M(G, K) \tilde{D}_{w}^{-1}\right\|_{\infty},
$$

with " $"$ " indicating minimum-phase, $\mathcal{R} \mathcal{H}_{\infty}$ versions of the original variables. The joint optimization for $K$ and $\tilde{D}$ is nonconvex and the global optimum may be hard to find. Instead, a sequence of convex optimizations is performed. This " $D-K$ iteration" is summarized below:

0 . An initial stabilizing controller $K$ is computed.

1. The convex problem $\inf _{D \in D} \bar{\sigma}\left(D_{z} M D_{w}^{-1}\right)$ is solved for a representative frequency grid, returning $\|M\|_{\bar{\mu}}$.

2. Over the grid, minimum-phase $\mathcal{R} \mathcal{H}_{\infty}$ transfer functions $\tilde{D}$ are fit in magnitude to $D(\omega)$ from step 1 .

3. The $\mathcal{H}_{\infty}$ optimization $\min _{K \in \mathcal{K}_{A}}\left\|\tilde{D}_{z} M(G, K) \tilde{D}_{w}^{-1}\right\|_{\infty}$ is performed and with the newly obtained $M$, step 1 is repeated.

For "optimal $D$ - $K$ iteration," this procedure is repeated until $\|M\|_{\bar{\mu}}$ in step 1 does not further decrease, while "suboptimal $D$ - $K$ iteration" stops once $\|M\|_{\bar{\mu}}<\gamma$, for given $\gamma$.

\section{The Input Output Selection Method}

Suppose optimal $D$ - $K$ iteration is performed for the full IO set including all candidate actuators and sensors and call the closed-loop $M^{\star}$. If $\left\|M^{\star}\right\|_{\bar{\mu}}<\gamma$, the full IO set is viable for the RP level $\gamma$ (recall, that in case $\left\|M^{\star}\right\|_{\bar{\mu}}>\gamma$ the full IO set may still be viable, since $\left.\left\|M^{\star}\right\|_{\mu} \leq\left\|M^{\star}\right\|_{\bar{\mu}}\right)$. The $D$-scales $D_{z}(\omega)$ and $D_{w}(\omega)$ corresponding to $M^{\star}$ are fit with minimum-phase $\mathcal{R} \mathcal{H}_{\infty}$ approximations, denoted by $\hat{D}_{z}$ and $\hat{D}_{w}$. An "estimate" $\left\|M^{\star}\right\|_{\hat{\mu}}$ for the $\mu$ upper bound $\left\|M^{\star}\right\|_{\bar{\mu}}$ is now defined as:

$$
\left\|M^{\star}\right\|_{\hat{\mu}}:=\left\|\hat{D}_{z} M^{\star} \hat{D}_{w}^{-1}\right\|_{\infty} .
$$

Comparing equations (3), (5), and (8), three different measures related with $M^{\star}$ can be computed, for which the following holds:

$$
\left\|M^{\star}\right\|_{\mu} \leq\left\|M^{\star}\right\|_{\bar{\mu}} \leq\left\|M^{\star}\right\|_{\hat{\mu}}
$$

The higher the order of $\hat{D}_{z}$ and $\hat{D}_{w}$, the better $\inf _{D \in D} \bar{\sigma}\left(D_{z} M^{\star} D_{w}^{-1}\right)$ and $\bar{\sigma}\left(\hat{D}_{z} M^{\star} \hat{D}_{w}^{-1}\right)$ will tend to match and the closer $\left\|M^{\star}\right\|_{\bar{\mu}}$ and $\left\|M^{\star}\right\|_{\hat{\mu}}$ will tend to be. The $D$-scale estimates $\hat{D}_{z}$ and $\hat{D}_{w}$ for the full IO set play a crucial role in the IO selection, the key idea of which is explained next.

An optimal $M$ for an IO set which is "almost as good" as the full IO set will have approximately the same dynamic behavior as $M^{\star}$, i.e., " $M^{\star}(s) \approx M(s)$." Moreover, 


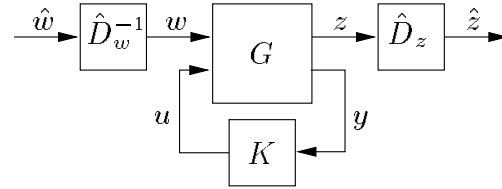

Figure 2: Extending $G$ with $D$-scale estimates

$\mu_{\Delta}(M) \approx \mu_{\Delta}\left(M^{\star}\right)$ and the $D$-scales for $M$ are roughly the same as those for $M^{\star}$. In that case, $\hat{D}_{z}$ and $\hat{D}_{w}$ are good estimates of the $D$-scales for $M$, provided $\hat{D}_{z}$ and $\hat{D}_{w}$ are good estimates of the $D$-scales for $M^{\star}$. If so, $\left\|\hat{D}_{z} M \hat{D}_{w}^{-1}\right\|_{\infty} \approx\|M\|_{\bar{\mu}}$, with $\|M\|_{\bar{\mu}}$ resulting from an optimal $D$ - $K$ iteration.

For each IO set, $G$ can be extended with the $D$-scales $\hat{D}_{z}$ and $\hat{D}_{w}^{-1}$ corresponding to $M^{\star}$, see Fig. 2. An estimate of the lowest achievable $\|M\|_{\bar{\mu}}$ value for each IO set is obtained by $\mathcal{H}_{\infty}$ optimization for each IO set's extended $G$ (like in the 3rd step of $D$ - $K$ iteration). It is emphasized, that this may be a conservative estimate, since $\hat{D}_{z}$ and $\hat{D}_{w}$ might not be the best approximations for other IO sets than the full one:

$$
\min _{K \in \mathcal{K}_{A}} \inf _{\tilde{D} \in \tilde{D}}\left\|\tilde{D}_{z} M \tilde{D}_{w}^{-1}\right\|_{\infty} \leq \min _{K \in \mathcal{K}_{A}}\left\|\hat{D}_{z} M \hat{D}_{w}^{-1}\right\|_{\infty}
$$

For the purpose of IO selection, the $D$-scale estimates $\hat{D}_{z}$ and $\hat{D}_{w}$ are used as follows:

IO Selection with $D$-Scale Estimates: Consider Fig. 2. For each candidate $\mathrm{IO}$ set, $G$ is scaled with $\hat{D}_{z}, \hat{D}_{w}^{-1}$ and it is tested if a stabilizing $K$ can be designed achieving $\|M\|_{\hat{\mu}}:=\left\|\hat{D}_{z} M \hat{D}_{w}^{-1}\right\|_{\infty}<\gamma$. Since this is a sufficient condition for $\|M\|_{\mu}<\gamma$, IO sets which pass are guaranteed to be viable for the RP level $\gamma$.

Unfortunately, due to sufficiency, IO sets which are viable might be eliminated. This shortcoming is expected to be more serious if the variables in $w$ and $z$ are directly linked with the selected inputs and outputs. First, this occurs for uncertainties in $\Delta_{u}$, which are related to the inputs or outputs, such as multiplicative or additive input or output uncertainties. This situation does not play a role for the application in this paper. Second, measurement noise (" $y$-noise") may be contained in $w$ and input weights (" $u$-weights") in $z$, as for the application. The presence of $y$-noise and $u$-weights is reflected in the last, normalized blocks of the $D$-scales. To investigate other IO sets than the full one, identity diagonal entries in $\hat{D}_{z}$ and $\hat{D}_{w}$ corresponding to non-considered inputs and outputs are skipped. It is expected, that the larger the $y$-noise and $u$ weights are, the less "close" $\hat{D}_{z}$ and $\hat{D}_{w}$ are to the optimal $D$-scales for each IO set, i.e., the gap in inequality (10) will be larger. After all, compared to other IO sets, the $D$ - $K$ iteration for the full one accounts for additional $u$-weights and $y$-noise. Section 5 briefly investigates this problem.

Essentially, the IO selection amounts to the same approach as in [9], where six conditions test the existence of a stabilizing controller achieving a desired closed-loop $\mathcal{H}_{\infty}$ norm for problems with unstructured $\Delta$. In fact, by extending $G$ with the $D$-scale estimates accounting for the structure in $\Delta$, an $\mathcal{H}_{\infty}$ control problem can be formulated again. During IO selection, the existence of a controller achieving RP is checked via the six conditions, but for the scaled plant in Fig. 2. For the sake of completeness, these conditions are summarized here.
For the state-space parameterization of $\mathcal{H}_{\infty}$ controllers, six "standard" assumptions on the state-space realization of the (unscaled) generalized plant $G$ are made. These must be satisfied for $\mathcal{H}_{\infty}$ controller design and for the IO selection; for details, see [3]. If these assumptions are met, they are also met for $G$ extended with $\hat{D}_{z}$ and $\hat{D}_{w}^{-1}$. To construct a stabilizing controller for the scaled plant achieving $\|M\|_{\hat{\mu}}<\gamma$, two Riccati equations must be solved, see [3] for their documentation. One of them is related with a state-feedback problem ("state-feedback Riccati"), the other with an observer problem ("observer Riccati"). The following lays the foundation for the IO selection method:

There exists a stabilizing controller achieving an RP level $\gamma$, if the following six conditions on the scaled generalized plant hold:

1. The direct feedthrough from $\hat{w}$ to $\hat{z}$ (see Fig. 2) is "not too large."

2,3. The two Hamiltonians associated with the statefeedback Riccati and the observer Riccati do not have $j \omega$-axis eigenvalues. Moreover, $\operatorname{Im}\left(\begin{array}{c}0_{n \times n} \\ I_{n}\end{array}\right)$ is complementary to each Hamiltonian's subspace corresponding to the stable eigenvalues.

4,5. The solutions $X_{\infty}$ and $Y_{\infty}$ to the state-feedback and observer Riccati respectively are positive semidefinite: $X_{\infty} \geq 0$ and $Y_{\infty} \geq 0$.

6. The spectral radius of the product $X_{\infty} Y_{\infty}$ is smaller than $\gamma^{2}: \rho\left(X_{\infty} Y_{\infty}\right)<\gamma^{2}$.

In the context of IO selection, these are called the "viability conditions," which are more precisely formulated in $[2,3]$. For the application in this paper, each IO set is subjected to the six conditions and as soon as one condition fails, the other are not checked. Ways to further improve efficiency are suggested in [8, Chapter 7]. For instance, subsets of nonviable $\mathrm{IO}$ sets need not be tested, since eliminating actuators and sensors will never yield better control.

\section{Active Suspension Control Problem}

The IO selection is investigated for an active suspension applied in the 4 Degree-Of-Freedom (DOF) vehicle model in Fig. 3. This model will not be elaborated here; upon request, a MATLAB file generating the model can be obtained from the first author. Two actuators $\left(u_{1}, u_{2}\right)$ placed between the axles and the tractor chassis are proposed as candidate inputs, while measurements of the suspension deflections $\left(y_{1}, y_{2}\right)$ and the chassis accelerations $\left(y_{3}, y_{4}\right)$ are suggested as candidate outputs. This yields 45 candidate IO sets, among which the $4 \times 2$ full IO set $y_{1} y_{2} y_{3} y_{4} / u_{1} u_{2}$ and eight $1 \times 1 \mathrm{IO}$ sets. This section provides the uncertainty characterizations and performance specifications, which are such that an IO set is termed viable if $\|M\|_{\mu}<1$.

Though various uncertain parameters play a role, only the uncertain semitrailer mass is incorporated in $\Delta_{u}$. This mass strongly depends on the transported cargo; the semitrailer may even be absent. Here, the mass is assumed to vary between the values for an empty and fully loaded semitrailer, with the nominal mass $M_{t}$ taken the mean of these two. This implies a $90 \%$ variation in the nominal value and $V_{1}=1$ and $W_{1}=0.9 M_{t}$ are used. Though this uncertainty 


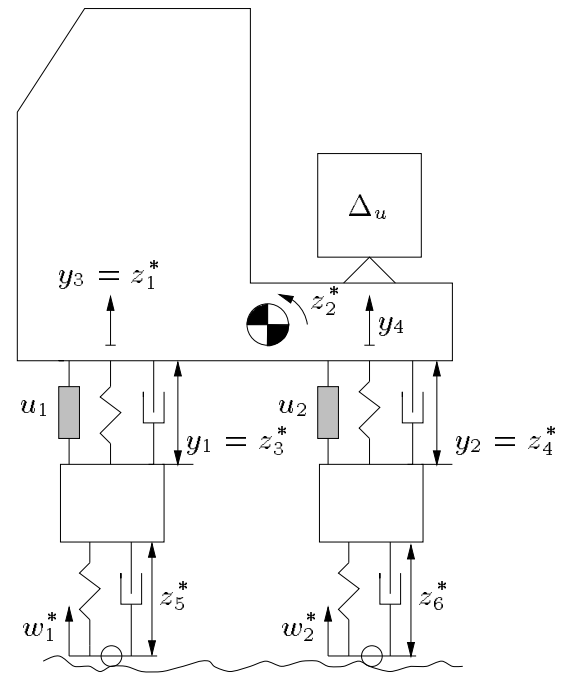

Figure 3: 4 DOF tractor-semitrailer combination

is a real parametric one, both the IO selection and the controller design in this paper account for the encompassing class of dynamic uncertainties, potentially introducing conservatism. Recall, that the $D$-scale corresponding to $\Delta_{p}$ is fixed at identity, so only a scalar estimate $\tilde{d}_{1}$ corresponding to $\Delta_{u}$ must be constructed for IO selection, see (6).

The exogenous input $w^{*}$ contains the excitation by the road surface, more specific, the derivatives of the road surface height $\left(w_{1}^{*}, w_{2}^{*}\right)$ and measurement noises $\left(w_{3}^{*}, \ldots, w_{6}^{*}\right)$. Assuming the road surface height to be lowpass-filtered white noise, the corresponding shaping filter in $V$ is chosen as:

$$
V_{2,3}=\frac{v_{0} s}{s / \omega_{0}+1}
$$

For a fair motorway and a forward vehicle speed of $25[\mathrm{~m} / \mathrm{s}]$, $\omega_{0}=2 \pi \cdot 0.25[\mathrm{rad} / \mathrm{s}]$ and $v_{0}=8.010^{-3}$ are representative choices.

The measurements are assumed to be disturbed with zeromean, white noises with an intensity of $\theta$ times their magnitude. These magnitudes are estimated by computing the $\mathcal{H}_{2}$ norms of the transfer function from $w^{*}$ to each measurement. For the road surface modeled by (11), this results in the following shaping filters:

$$
\begin{aligned}
V_{4,5} & =3.310^{-3} \theta & & \text { (suspension deflections), } \\
V_{6} & =5.710^{-1} \theta & & \text { (front chassis acceleration), } \\
V_{7} & =8.710^{-1} \theta & & \text { (rear chassis acceleration). }
\end{aligned}
$$

Parameter $\theta$ can be interpreted as the error fraction in the measured variables. Here, $\theta=0.05$.

Four main design goals in $z^{*}$ are distinguished [4]. First, good driver comfort must be guaranteed (as well as cargo comfort, but this is not considered here). The tractor's vertical acceleration at the front $z_{1}^{*}$ and the rotational acceleration $z_{2}^{*}$ quantitatively represent driver comfort. The choice of filters in $W$ is based on human sensitivity plots for accelerations provided in [4]. The sensitivity contour for $z_{1}^{*}$ is approximated by the magnitude of $W_{2}$ :

$$
W_{2}=\rho_{1} \omega_{1}^{2} \frac{s / \omega_{2}+w_{10}}{s^{2}+2 \zeta \omega_{1} s+\omega_{1}^{2}},
$$

with $\rho_{1}=1, w_{10}=0.4, \zeta=1, \omega_{1}=2 \pi \cdot 10[\mathrm{rad} / \mathrm{s}]$, and $\omega_{2}=2 \pi \cdot 5[\mathrm{rad} / \mathrm{s}]$. For the rotational acceleration, the following weighting filter is used:

$$
W_{3}=\rho_{2} \frac{w_{20}}{s / \omega_{3}+1}
$$

with $\rho_{2}=1, w_{2_{0}}=1$, and $\omega_{3}=2 \pi \cdot 2[\mathrm{rad} / \mathrm{s}]$.

The second and third design goal in $z^{*}$ are limiting the suspension deflections (due to space limitations) and the tire deflections (for good handling and minimum road surface damage) respectively. For these design goals, one is actually interested in restricting the $\mathcal{L}_{1}$ norm, so suitable weights in the $\mathcal{H}_{\infty}$ norm setting are hard to give. Here, the front and rear weights are chosen equal and constant:

$$
\begin{aligned}
W_{4,5}=\rho_{3}=90 & \text { (suspension deflections), } \\
W_{6,7}=\rho_{4}=330 & \text { (tire deflections). }
\end{aligned}
$$

Though the suspension and tire deflection limits will normally not be exceeded for stochastic road surfaces [4], this might happen for deterministic ones. With the chosen $\rho_{3}$ and $\rho_{4}$ values, $\mathcal{H}_{\infty}$ optimizations for the full $\mathrm{IO}$ set and $\Delta_{u}=0$ show, that the design goals accelerations, suspension deflections, and tire deflections are "equally important," i.e., the same $\|M\|_{\infty}$ values are achieved for these subproblems. Also, the closed-loop shows acceptable behavior for a special class of road surfaces [8].

Finally, $u$-weights are formulated. In general, the bandwidth of actuators is limited and high-frequency inputs cannot be realized. Therefore, the following bi-proper weighting filters for $z_{7}^{*}=u_{1}$ and $z_{8}^{*}=u_{2}$ are used:

$$
W_{8,9}=\rho_{5} \frac{s / \omega_{4}+1}{s / \omega_{5}+1} \text {. }
$$

It is assumed, that the bandwidth of the actuators is $5[\mathrm{~Hz}]$, i.e., $\omega_{4}=2 \pi \cdot 5[\mathrm{rad} / \mathrm{s}]$. Furthermore, $\omega_{5}=100 \omega_{4}$ ( $" \omega_{5} \gg \omega_{4}$ ") and $\rho_{5}=510^{-6}$, so $u$-weights are neither negligible nor dominant. For more detailed motivations of the specifications, see [8].

\section{Illustrative Application}

This section first discusses the results of $\mu$-syntheses and $\mathcal{H}_{\infty}$ optimizations of (scaled) plants for some typical IO sets. The aim is to acquire insight on the importance of each sensor and actuator and to illustrate some issues from Section 3. Second, the outcome of the IO selection with the 45 candidate IO sets is discussed and compared with the outcome from suboptimal $\mu$-synthesis. The MAT$\mathrm{LAB} \mu$-Toolbox [1] is employed for controller design.

\subsection{Controller Design for Typical IO Sets}

Preceding the IO selection, some detailed studies are performed for the nine typical IO sets in Table 1. Comparing the $\|M\|_{\infty}$ values from $\mathcal{H}_{\infty}$ optimizations for Nominal Performance (NP) (i.e., in the absence of $\Delta_{u}$ ) and the $\|M\|_{\bar{\mu}}$ values from optimal $D$ - $K$ iterations, the differences are quite large, indicating a large performance degradation due to the uncertainty. Once more, it is emphasized that $\mu$-synthesis accounting for real uncertainties may yield smaller $\mu$ values.

Comparing the $\|M\|_{\bar{\mu}}$ values for IO sets 1,2 , and 3 , it is observed, that IO set 2 employing the single front actuator 
Table 1: Optimal controller design results for the unscaled $G$ and influence of the $D$-scale estimate's order on the optimal $\|M\|_{\bar{\mu}}$ of the scaled $G$ for some typical IO sets.

\begin{tabular}{cccccccccc}
\hline IO set & $\begin{array}{c}\|M\|_{\infty} \\
(\mathrm{NP})\end{array}$ & $\begin{array}{c}\|M\|_{\bar{\mu}} \\
(\mathrm{RP})\end{array}$ & \multicolumn{5}{c}{$\begin{array}{c}\|M\|_{\hat{\mu}} \\
(\mathrm{RP})\end{array}$} \\
\cline { 5 - 11 } & & & & \multicolumn{5}{c}{$D$-scale order } \\
\cline { 5 - 11 } & & & & $\times$ & 0 & 1 & 2 & 3 \\
\hline 1 & $y_{1} y_{2} y_{3} y_{4} / u_{1} u_{2}$ & 0.15 & 0.76 & 189.96 & 3.38 & 0.76 & 0.76 & 0.75 \\
2 & $y_{1} y_{2} y_{3} y_{4} / u_{1}$ & 0.17 & 1.06 & 190.17 & 12.40 & 1.06 & 1.07 & 1.08 \\
3 & $y_{1} y_{2} y_{3} y_{4} / u_{2}$ & 0.27 & 0.76 & 189.96 & 3.58 & 0.76 & 0.76 & 0.75 \\
4 & $y_{1} y_{2} / u_{1} u_{2}$ & 0.15 & 0.77 & 435.63 & 5.66 & 0.77 & 0.77 & 0.77 \\
5 & $y_{3} y_{4} / u_{1} u_{2}$ & 0.16 & 0.76 & 207.50 & 3.38 & 0.76 & 0.76 & 0.76 \\
6 & $y_{1} / u_{1} u_{2}$ & 0.16 & 1.01 & 972.46 & 12.48 & 1.16 & 1.13 & 1.07 \\
7 & $y_{2} / u_{1} u_{2}$ & 0.27 & 0.77 & 436.08 & 5.66 & 0.77 & 0.77 & 0.77 \\
8 & $y_{3} / u_{1} u_{2}$ & 0.17 & 0.83 & 946.19 & 12.16 & 1.18 & 1.13 & 1.06 \\
9 & $y_{4} / u_{1} u_{2}$ & 0.28 & 0.77 & 288.35 & 3.70 & 0.76 & 0.76 & 0.76 \\
\hline
\end{tabular}

$u_{1}$ is nonviable. This implies, that also the other $14 \mathrm{IO}$ sets based on the single $u_{1}$ are nonviable, since eliminating sensors will never improve control. IO set 1 and 3 are equally best for RP. Apparently, adding $u_{1}$ is only beneficial for NP. This is due to $q$ dominating $z^{*}$ in the minimization of $\|M\|_{\bar{\mu}}$ and $u_{1}$ being useful for $z^{*}$, but not as much for $q$ : studying the open-loop transfer functions from $u_{1}$ to $q$ and from $u_{2}$ to $q$ (not depicted), it appears that the gain from $u_{2}$ to $q$ is largest, so it takes less effort to affect $q$ with $u_{2}$ than with $u_{1}$. In summary, if RP is the focus the rear actuator is preferred to the front one.

The NP and RP results for IO set 4 and 5 are the same, i.e., a set of two displacement measurements is equally best as a set of two acceleration measurements. In case of RP and one sensor (IO sets 6-9), the acceleration at the front $y_{3}$ is preferred to the suspension deflection at the front $y_{1}$. The IO set based on the single $y_{1}$ is not even viable for RP (so neither will be $y_{1} / u_{1}$ and $y_{1} / u_{2}$, since eliminating actuators will not improve control). The rear displacement and acceleration measurements $y_{2}$ and $y_{4}$ are equally best and better (for RP) than the front measurements. Studying the open-loop transfer functions from the front road input $w_{1}$ to $q$ and the rear road input $w_{2}$ to $q$, the gain from $w_{2}$ to $q$ is largest. This may be the reason why rear sensors are preferred to front ones (as well as it may be a second reason for preference of $u_{2}$ to $u_{1}$ ).

Table 1 also depicts $\|M\|_{\hat{\mu}}$ values resulting from $\mathcal{H}_{\infty}$ optimizations for the generalized plants scaled with the $D$ scale estimate constructed for the full IO set. The column marked with " $\times$ " depicts the results for identity $D$-scale estimates, or, equivalently, the results for conventional $\mathcal{H}_{\infty}$ optimizations. Comparing the $\|M\|_{\hat{\mu}}$ values with $\|M\|_{\bar{\mu}}$ illustrates inequality (10) (sometimes $\|M\|_{\hat{\mu}}=$ $\|M\|_{\bar{\mu}}-0.01$, which is only due to the limited numerical accuracy in the $D$ - $K$ iteration). To generate $\hat{D}$, two functions from the $\mu$-Toolbox [1] are used. In case of constant $D$-scale estimates, musynflp is used, since musynf it returns incorrect estimates; for first and higher order estimates musynfit is used, due to numerical problems with high order musynflp estimates. The estimates are computed for a grid of 201 logarithmically spaced frequency points between $10^{-2}$ and $10^{4}[\mathrm{rad} / \mathrm{s}]$. Figure 4 shows the frequency dependent $D$-scale and four approximations. Especially in the region between $10^{1}$ and $10^{2}[\mathrm{rad} / \mathrm{s}$ ] where $\mu$ peaks, the first and higher order fits are reasonable.

From Table 1, the conservatism of the $\mathcal{H}_{\infty}$ design is obvious if the $\|M\|_{\hat{\mu}}$ values for identity $D$-scales and $\|M\|_{\bar{\mu}}$ are

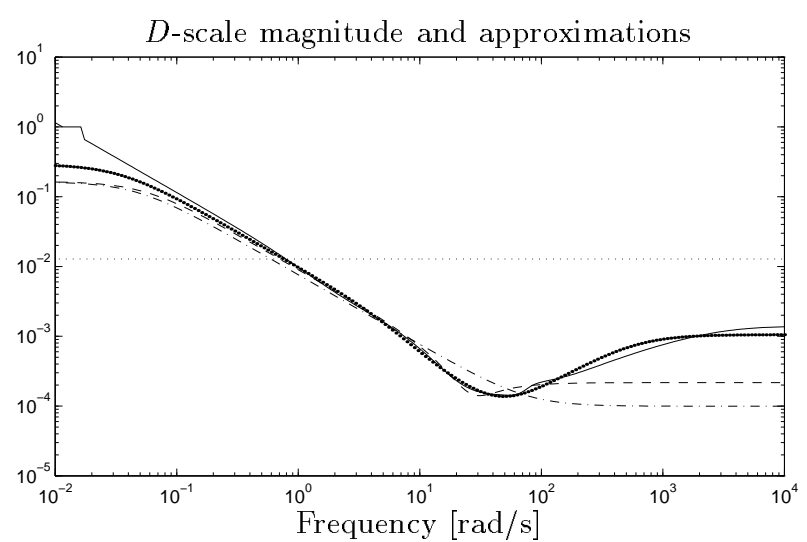

Figure 4: The frequency dependent $D$-scale for the full IO set $(-)$ and some approximations: 0th order $(.) ;$.1 st order (-.); 2nd order (--); 3rd order (...)

Table 2: Results with the IO selection procedure and with suboptimal $D$ - $K$ iteration for 45 candidate IO sets

\begin{tabular}{ccccc}
\hline & \multicolumn{2}{c}{ IO selection } & \multicolumn{2}{c}{$D$ - $K$ iteration } \\
\hline$\hat{D}$-order & Viable IO sets & CPU $[\mathrm{s}]$ & Viable IO sets & CPU $[\mathrm{s}]$ \\
\hline 0 & 0 & 93 & & \\
1 & 26 & 170 & 28 & $8.3410^{3}$ \\
2 & 26 & 209 & 28 & $8.7810^{3}$ \\
3 & 26 & 253 & 28 & $10.3410^{3}$ \\
\hline
\end{tabular}

compared. By invoking the $D$-scale estimates accounting for structured $\Delta$, this conservatism is reduced. For constant estimates, the gap between $\|M\|_{\bar{\mu}}$ and $\|M\|_{\hat{\mu}}$ is still large. If IO selection were performed with $\gamma=1$, IO sets would incorrectly be rejected. For first order estimates, the results are considerably better: $\|M\|_{\hat{\mu}}=\|M\|_{\bar{\mu}}$, except for IO set 6 and 8. Further increasing the order improves the results for these two IO sets. However, for IO set 2, the results get slightly worse, as they do for IO set 6 and 8 in case of 4 th and 5 th order estimates (not depicted). So, higher order estimates not always give less conservative results: if the optimal $D$-scales for an IO set considerably differ from those for the full IO set, improved $D$-scale estimates for the full IO set need not be closer to the optimal ones.

The studies above have also been performed for the case with negligibly small $y$-noise and $u$-weights (they must be nonzero to meet the $\mathcal{H}_{\infty}$ standard assumptions). In Section 3 , it is conjectured, that the gap in inequality (10) is now smaller. Indeed, for IO set 6 and 8 the results are better: for a third order $D$-scale, the gaps are 0.02 instead of 0.06 and 0.12 compared to 0.23 respectively. However, the results for IO set 2 are worse: for a third order $D$-scale, the gap is 0.07 instead of 0.02 . Comparing the $\|M\|_{\bar{\mu}}$ values for the cases with and without $y$-noise and $u$-weights, these appear to be approximately the same. Hence, the effect of $y$-noise and $u$-weights is relatively small, which may be the reason that the conjecture is not clearly supported by the nine typical IO sets for the example.

\subsection{IO Selection Results}

During IO selection, all 45 candidate IO sets are checked for an RP level $\gamma=1$. For $D$-scale orders $0-3$, Table 2 shows the number of accepted IO sets. As expected, IO selection based on zeroth order estimates eliminates all 45 candidates. For first till third order $D$-scales, always the same 26 
IO sets are viable. The $15 \mathrm{IO}$ sets employing only the front actuator $u_{1}$ and the IO sets $y_{1} / \boldsymbol{u}_{2}, y_{1} / \boldsymbol{u}_{1} \boldsymbol{u}_{2}$ employing only the front suspension deflection measurement are indeed nonviable, as predicted from Table 1. Apart from these 17 correctly rejected IO sets, $y_{3} / u_{2}$ and $y_{3} / u_{1} u_{2}$ (both $\left.\|M\|_{\bar{\mu}}=0.83\right)$ are incorrectly rejected. The IO sets with the least number of actuators and sensors which the IO selection accepts are $y_{2} / \boldsymbol{u}_{2}$ and $y_{4} / \boldsymbol{u}_{2}$ (both $\|M\|_{\bar{\mu}}=0.77$ ) and so these are preferable in the sense of the IO selection goal stated in the Introduction. IO selection was also performed "without" $y$-noise and $u$-weights, in which case no IO sets were incorrectly eliminated.

It is expected, that IO selection based on higher order $D$ scales tends to give less conservative results in general. However, as discussed in the previous section this need not be true. Besides, higher orders give rise to more computational effort, since the order of $G$ increases with twice the $D$-scale order. In turn, this yields more complex viability conditions. Table 2 compares the CPU times on a SUN Sparc Station 10/31 for various $D$-scale orders. This is done for the IO selection (with the $D$-scale estimates computed beforehand) and for suboptimal $\mu$-syntheses. In the latter case, $D$ - $K$ iterations are performed for all 45 IO sets, where the $D$-scales are fit for 101 frequency points logarithmically spaced between $10^{-1}$ and $10^{3}[\mathrm{rad} / \mathrm{s}]$ (see second step in $D$ - $K$ iteration). Note, that the number of frequency points also greatly affects the computational effort. For zeroth order approximations, no results are depicted, because the $D-K$ iteration did not converge, or converged to the wrong $\|M\|_{\bar{\mu}}$ value. For higher order approximations, the $D$ - $K$ iteration is stopped as soon as $\|M\|_{\bar{\mu}}<1$, or if $\|M\|_{\bar{\mu}}$ does not reduce further than 0.01 (always within five steps).

From Table 2, it is obvious, that low order $D$-scales are preferred for efficiency. For the particular problem considered here, there is no need to use second or third order $D$-scales. So, a "guideline" for choosing appropriate orders of $D$-scale estimates for IO selection is highly desirable, but currently lacking. Clearly, IO selection via suboptimal $D-K$ iteration is considerably less efficient; especially for a large number of candidate IO sets, this is a major disadvantage. On the other hand, suboptimal $D$ - $K$ iterations do not eliminate viable IO sets. It is emphasized again, that the efficiency of both approaches could be improved significantly by using a smarter implementation, which avoids checking all IO sets (see [8, Chapter 7]).

\section{Discussion and Future Research}

A new IO selection approach was studied, aimed at eliminating IO sets for which a desired RP level cannot be achieved. Contrary to many other methods [10], the new one is not restricted to IO sets with the same number of actuators and sensors, it separates the measured and controlled variables, and it allows for quantitative performance specifications and uncertainty characterizations for any frequency range of special interest.

A major shortcoming is, that the new method may incorrectly reject IO sets, as illustrated by the example. The gap between the employed sufficient condition for an IO set to achieve the RP level $\gamma\left(\min _{K}\left\|\hat{D}_{z} M \hat{D}_{w}^{-1}\right\|_{\infty}<\gamma\right)$ and the necessary and sufficient condition $\left(\min _{K}\|M\|_{\mu}<\gamma\right)$ may be large, which is due to various sources. First, IO selec- tion uses the $\mu$ upper bound: $\left\|M^{\star}\right\|_{\bar{\mu}} \geq\left\|M^{\star}\right\|_{\mu}$, with $M^{\star}$ the full IO set's optimal closed-loop. Fortunately, in general $\left\|M^{\star}\right\|_{\bar{\mu}} \approx\left\|M^{\star}\right\|_{\mu}$. Second, low order $D$-scale estimates may give crude approximations: $\left\|\hat{D}_{z} M^{\star} \hat{D}_{w}^{-1}\right\|_{\infty} \geq\left\|M^{\star}\right\|_{\bar{\mu}}$. Further investigation is needed to find out how to choose the order so the IO selection is both efficient and effective. Third, the $D$-scale estimate corresponding to $M^{\star}$ may be worse for other IO sets. One reason is, that compared to the other IO sets the $D$-scale estimate from the full IO set accounts for additional control objectives if $y$-noise and $u$ weights are included (via $\Delta_{p}$ ), or if uncertainty associated with $u$ or $y$ is present (via $\Delta_{u}$ ). In order to draw more general conclusions on the practical aspects of this conservatism, the new IO selection should also be applied to the active suspension control problem with different uncertainty sources and to other examples.

A necessity-based $\mathrm{IO}$ selection method aimed at the same goal is proposed in [5] and further studied in [7]; in [8], it is compared with the $D$-scale method. The method from [5] indeed eliminates conservatism, but applications show, that it is less efficient than the method studied here and that nonviable IO sets may be accepted. Another idea for IO selection is currently studied, which is essentially as follows. Based on the $\mu$ definition (4), a $\Delta$ making $I-M^{\star} \Delta$ singular across the frequency grid is computed. By replacing the $\Delta_{u}$-part of this $\Delta$ with an $\mathcal{R} \mathcal{H}_{\infty}$ representation and absorbing this into the plant, the (structured) uncertainty block is eliminated. For the remaining, unstructured performance block $\Delta_{p}$, the viability conditions from Section 3 can be used again.

\section{References}

[1] G. J. Balas, J. C. Doyle, K. Glover, A. Packard, and R. Smith. $\mu$-Analysis and synthesis toolbox. The MathWorks, Natick, MA, USA, 1991. version 2.0.

[2] J. C. Doyle, K. Glover, P. P. Khargonekar, and B. A. Francis. State-space solutions to standard $\mathcal{H}_{2}$ and $\mathcal{H}_{\infty}$ control problems. IEEE Tr. Aut. Control, 34(8):831-846, 1989.

[3] K. Glover and J. C. Doyle. State-space formulae for all stabilizing controllers that satisfy an $\mathcal{H}_{\infty}$-norm bound and relations to risk sensitivity. Systems \& Control Letters, 11:167-172, 1988. [4] B. de Jager. Multiobjective suspension control problem. In Proc. of the 34th IEEE Conf. on Decision \& Control, volume 4, pages 3652-3657, New Orleans, LA, 1995.

[5] J. H. Lee, R. D. Braatz, M. Morari, and A. Packard. Screening tools for robust control structure selection. Automatica, 31(2):229-235, 1995.

[6] A. Packard and J. C. Doyle. The complex structured singular value. Automatica, 29(1):71-109, 1993.

[7] P. Philips, M. van de Wal, and B. de Jager. Selection of sensors and actuators based on a necessary condition for robust performance. In Proc. of the European Control Conf., Brussels, Belgium, 1997.

[8] M. van de Wal. Input output selection based on robust performance: An active suspension application. Technical Report WFW 96.048, Fac. of Mechanical Engineering, Eindhoven University of Technology, 1996.

[9] M. van de Wal and B. de Jager. Selection of sensors and actuators for an active suspension control problem. In Proc. of the IEEE Int. Conf. on Control Applications, Dearborn, MI, 1996.

[10] M. van de Wal and B. de Jager. Control structure design: A survey. In Proc. of the American Control Conference, volume 1, pages 225-229, Seattle, WA, 1995.

[11] K. Zhou, J. C. Doyle, and K. Glover. Robust and optimal control. Prentice Hall, Upper Saddle River, NJ, 1996. 